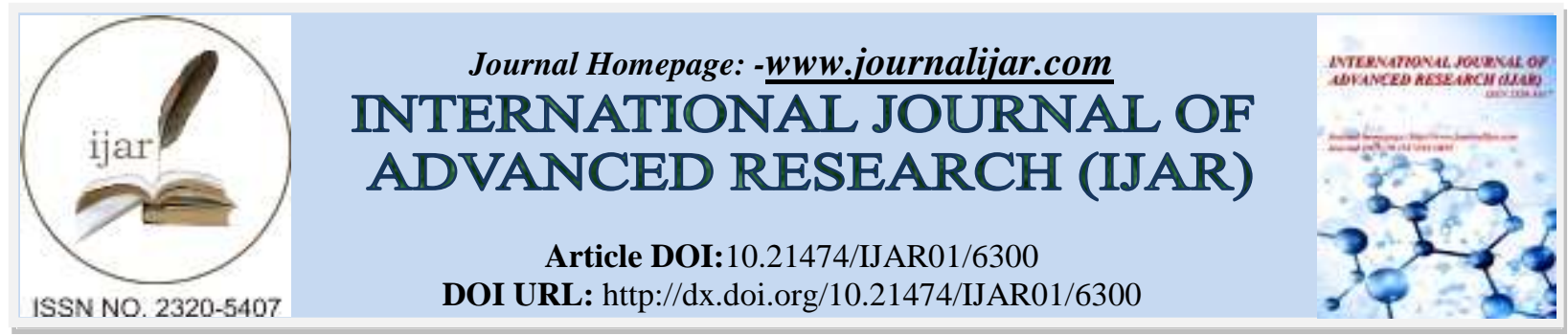

RESEARCH ARTICLE

\title{
STUDIES ON HEADING DATE FOR SYNCHRONIZATION AND HYBRIDIZATION OF FIVE RICE VARIETIES UNDER SHORT DAY LENGTH AND NEUTRAL DAY LENGTH.
}

ZinWai Maw ${ }^{1}$ and Irie Kenji ${ }^{2}$.

1. M.Sc. Student of Laboratory of Tropical Crop Science, Department of International Agricultural Development, Tokyo University of Agriculture, Tokyo, Japan.

2. Department of International Agricultural Development, Tokyo University of Agriculture, Tokyo, Japan.

\section{Manuscript Info}

\section{Manuscript History}

Received: 14 November 2017

Final Accepted: 16 December 2017

Published: January 2018

\section{Key words:-}

Paw San Hmwe, F1 hybrids, Synchronization, Hybridization, Short day length, neutral day length.

\section{Abstract}

Rice is a short day plant and flowering is promoted in short day conditions and suppressed in long day condition. Short-day condition allows the production of plants in different flowering stages which greatly facilitates synchronous flowering for varieties that differ in their flowering time. The heading date for synchronization of Paw San Hmwe (PSM) and other four indica rice varieties were studied under short day length and neutral day length condition and F1 seed set was observed from hybridization of PSM and indica rice varieties by hot water emasculation method. Results showed days to heading of PSM and other four indica varieties in short day length was earlier than those in neutral day length.Among the tested varieties, PSM was highly sensitive to high temperature and photoperiod in neutral day length due to not only flowering in short day photoperiod condition but also photoperiod sensitive and late cultivar. The present study revealed that although five rice varieties were cultivated to adjust synchronization under neutral day length and short day length for hybridization, synchronization for PSM and other four indica rice varieties was not found in both conditions because it might be different genetic variation on the tested varieties and sowing date of tested varieties was same under neutral day length and short day length condition. Synchronization was found in short day length and cultivation in concrete tank of net house. PSM from short day length condition was used as female parent for hybridization because it was synchronized with the other three indica varieties from second time cultivation in concrete tank of net house. The F1 seed set percent of PSM x IR 24 was higher than the other tested crosses and collected two F1 hybrids (PSM x IR 24 and PSM x Thaichung 65). Therefore, short day length condition ( 9 hour light and 15 hour dark) could be used for synchronization and hybridization on rice genotypes especially PSM (photoperiod sensitive variety) compared to neutral day length condition. The F1 hybrids (PSM x IR 24 and PSM x Thaichung 65) from this study should be used to get new rice varieties through several techniques. 


\section{Introduction:-}

Rice is one of the world's most important staple crops. In Asia, rice supplies 30-80\% of the daily calories consumed (Nareiso and Hossian, 2002). Most of rice growing areas in Myanmar are located in delta region (Ayeyarwaddy Division, Bago Division, Yangon Division, Mon State) and coastal region (Rakhine State, Taninthari Division). The major extension of rice cultivation took place mostly on that area because their hydromorphic nature was exclusively suited for rice (IRRI, 1976). The climatic condition of these areas is tropical wet due to monsoon from the Bay of Bengal. Myanmar has to increase rice production through area expansion, yield increasing per unit area and crop intensification. The total sown area of paddy was 8.1 million hectares, compressing 6.8 million hectares under monsoon paddy, 1.3 million hectares under summer paddy and average yields was 3.9MT ha-1 (MOAI 2014). Production and provision of high-yielding and quality seeds for paddy have been undertaking throughout the country for the benefit of farmers and for the varieties with high yielding and adaptable to different environments (MOAI 2014).

Among rice varieties; Myanmar local quality rice, Paw San Hmwe (PSM) was traditionally grown in rain- fed lowland of water logged area or marginal land. Cultivated area in 2007 was 449487 hectare, representing $6.5 \%$ of total rice area in Myanmar. Paw San Hmwe rice has been cultivated in many areas of Myanmar for long time. The names of Paw San rice vary by location and official names. Paw San Hmwe is the market name for two varieties of Paw San rice, namely paw san gyi known as Shwebo Paw San, and Paw San Yin. The Shwebo Paw San is photoperiod sensitive and retains its aroma for up to 2 years with proper harvesting and storage, while Paw San Yin is non-photoperiod sensitive and possesses a strong aroma that fades after 5-6 months (Win.U.K, 1991).Strong aroma, good taste and its elongation are the key characteristics of PSM rice. Paw San Hmwe is an aromatic rice variety and it was awarded World's best rice at the World Rice Conference 2011 held in Ho Chi Minh, Vietnam. It is well adapted to the particular local environmental conditions especially in delta region of Myanmar (Thein, 2011). It has the potential to become famous in the international market soon; it is challenge to develop superior quality rice in international rice market. But yield per hectare is relatively low (3.1 -3.6 MT/ha) because it is photoperiod sensitive and it can flower under short day condition. Therefore, it is difficult to know its heading date and also difficult to be synchronization when hybridized with other improved varieties. In this case, we should create short day length condition for synchronization and hybridization. Short days were most effective on tillering plants which have 7 to 9 leaves on certain Japanese varieties of paddy (Rjabov, 1941). Rice is a short day plant and flowering is promoted in short day conditions and suppressed in long day condition (Garner and Allard, 1920). Heading date is very important in rice cultivation. Zou et al, 2008 reported that the heading rate of LY 29 is $21 \%$ in the short day length and high temperature conditions and $13.6 \%$ in neutral day length and high temperature conditions. Heading date of some Indica and Japonica varieties was strongly influenced by their photoperiod sensitive gene in short day length (Zhou.et.al, 2011). Short-day condition allows the production of plants in different flowering stages which greatly facilitates synchronous flowering for varieties that differ in their flowering time (Song Yuanli.et.al, 2012). Short day treatment could not only reduce plant height but also shorten 50\% flowering (Ganashen and Whittingtun, 1976 and Craufurd et.al., 2003). However, the complete information had not been provided on PSM to be synchronized and hybridzed with other improved indica varieties. For these reasons, synchronization and hybridization were conducted by applying short day and neutral day treatment and the present study was done to study heading date for synchronization of Paw San Hmwe (PSM) and other four indica rice varieties under short day length and neutral day length condition and to observe F1 seed set from hybridization of PSM and indica rice varieties by hot water emasculation method.

\section{Materials and Methods:-}

Japonica and photoperiod sensitive variety, Paw San Hmwe (PSM) and four indica varieties, IR 24, Thaichung 65, IR 64 and culture 340 ( photoperiod insensitive varieties) were grown in short day length and neutral day condition. Paw San Hmwe (PSM) was introduced from seed bank of Myanmar and four indica rice varieties were collected from Tropical Crop Science Laboratory, Tokyo University of Agriculture. This study was conducted in a greenhouse at the Tokyo University of Agriculture in Japan. The experiment was set from June 2016 to November 2016. The experiment was laid out in Randomized Complete Block Design (RCB) with three replications. In short day length and neutral day length conditions, one pot is one replication and two seedlings per pot were grown respectively.In the short day condition, short day treatment was given to the seedling after transplanting for 30 days and neutral day was given as in green house condition. The temperature in the short day length condition was controlled to get rapid heading at $31 \mathrm{C}$ and the photoperiod was 9 hour light and 15 hour dark. The temperature of rice plants which are 
under neutral day length condition in green house were as in greenhouse condition and photoperiod was also as in natural.

\section{Heading date investigation:-}

The heading date was recorded for each variety when the first developing panicle emerged about $1 \mathrm{~cm}$ above the leaf sheath of the flag leaf and checked up every day. The mean days from transplanting to heading of each variety were taken as a heading date.

\section{Hybridization of PSM and indica rice varieties:-}

Paw San Hmwe (PSM) (Japonica) and IR 24, IR 64, Thaichung 65 and Culture 340 (Indica) were sown three times with one month intervals in concrete tank of net house for synchronization with PSM from neutral day length and short day length condition. First time sowing date in concrete tank was 2016, June, 8th to 2016, October, 20 ${ }^{\text {th }}$, second time was 2016 , July, $8^{\text {th }}$ to 2016 , November, $20^{\text {th }}$ and third time was 2016 , August, $8^{\text {th }}$ to 2016 , December, $20^{\text {th }}$ (Plate. 3.2). Two seedlings per hill were grown and spacing was $25 \mathrm{~cm}$ between row and $25 \mathrm{~cm}$ between plants were grown in a concrete tank of net house. PSM was used as female parent and hybridized with indica rice varieties. Hybridization was done in a greenhouse on October 2016. F1 seeds of (PSM x IR24) and (PSMx Thaichung 65) were collected on November 2016.

\section{Hot water emasculation method:-}

In this study, panicle emasculation with hot water method (Matsubayashi et al., 1965) was used for hybridization. After removing flag leaves, Panicles were dipped into water tank contained hot water at 43.C for 7 minutes (Plate 3.2.3) . The pollen could be killed by hot water and could create as female parent. After hot water emasculation, pollination was done by using several panicles for each varieties. Number of florets used for emasculation, number of panicles used for pollination, number of seed set and F1 seed set percent (\%) were recorded.

$$
\text { F1seed set }(\%)=\frac{\text { Number of seed set }}{\text { Number of florets used for emasculation }} \times 100
$$

\section{Data analysis:-}

The data were subjected to analysis of variance and mean separation was Tukey's Studentized Range (HSD) test using JMP 5 system software at 5\% level. Data calculation was done by microsoft excel and data was expressed in percentage value for F1 seed set.

\section{Results and Discussion:-}

Effect of plant height, no. of tiller per hill, days to heading of five rice varieties under neutral day length condition:-

Mean numbers of plant height, no. of tiller per hill and days to heading of five rice varieties grown under neutral day length condition in greenhouse were presented Table 1.1.There were significant differences among the tested varieties for mean numbers of plant height on five rice varieties. The shortest plant height was observed in IR 64 $(71.18 \mathrm{~cm})$ and followed by Thaichung $65(87.81 \mathrm{~cm})$, IR24 $(92.58 \mathrm{~cm})$ and culture $340(94.68 \mathrm{~cm})$ while the tallest plant height on Paw San Hmwe (PSM) $(114.25 \mathrm{~cm})$. According to the results, it could be assume that Paw San Hmwe (PSM) showed a remarked increase in plant height $(114.25 \mathrm{~cm})$ due to prolonged vegetative period while IR 64 showed a considerable decrease in plant height $(71.18 \mathrm{~cm})$ due to reduced vegetative phase. Similar results reported that the increase of plant height was an indication of changing vegetative to reproductive phase of crop growth (Krishnan et al., 2011).

Mean number of tiller (no.) in neutral day length condition for five rice varieties was significantly different. The number of tillers hill-1 were counted minimum to maximum. The maximum mean number of effective tillers hill-1 was observed in IR 64 (11.67) which was significantly higher than the other tested varieties. According to the data, the maximum mean number of effective tillers hill was found in IR 64 (11.67) seems be to due to their genetic variations.

Days to heading showed significant difference among the tested varieties ranged between 66-143 days. Days to heading was counted from days to sowing to heading. The earliest heading variety was found in culture 340 (66 days) followed by the other tested varieties and the latest one was obtained in Paw San Hmwe (143 days). In my study, Paw San Hmwe was the latest variety among the tested variety because there was high temperature and high 
light intensity during September to October, 2016 in Tokyo, Japan. It might be due to containing photoperiod sensitive gene as well as it was sensitive to photoperiod. Abdul, 1969 studied that high temperature and long photoperiod delayed heading.

Table 1.1:-Plant height, number of tiller per hill and days to heading under neutral day length condition in greenhouse at the Tokyo University of Agriculture

\begin{tabular}{|l|l|l|l|}
\hline Varieties & $\begin{array}{l}\text { Plant height } \\
\text { (Mean } \pm \text { SE) }\end{array}$ & $\begin{array}{l}\text { No. of tiller } \\
\text { per hill } \\
\text { Mean } \pm \text { SE) }\end{array}$ & $\begin{array}{l}\text { Days to } \\
\text { Heading } \\
\text { (Mean } \pm \text { SE) }\end{array}$ \\
\hline PSM & $114.25 \pm 1.26 \mathrm{a}$ & $7.83 \pm 0.44 \mathrm{~b}$ & $143 \pm 0.33 \mathrm{a}$ \\
\hline Culture340 & $94.68 \pm 2.66 \mathrm{~b}$ & $4.16 \pm 0.08 \mathrm{c}$ & $66 \pm 1.20 \mathrm{~d}$ \\
\hline IR 24 & $92.58 \pm 0.06 \mathrm{~b}$ & $8.00 \pm 0.25 \mathrm{~b}$ & $94 \pm 0.00 \mathrm{~b}$ \\
\hline Taichung65 & $87.81 \pm 1.86 \mathrm{~b}$ & $5.75 \pm 0.52 \mathrm{c}$ & $90 \pm 0.00 \mathrm{c}$ \\
\hline IR 64 & $71.18 \pm 1.54 \mathrm{c}$ & $11.66 \pm 0.58 \mathrm{a}$ & $96 \pm 0.00 \mathrm{~b}$ \\
\hline Pr>F & $<0.0001$ & $<0.0001$ & $<0.0001$ \\
\hline CV\% & 3.2 & 9.6 & 1.2 \\
\hline
\end{tabular}

Means followed by the same letter are not significantly different at 5\% level by Tukey’sStudentized Range (HSD) test

Effect of plant height, no. of tiller per hill, days to heading of five rice varieties under short day length condition:-

Mean plant height, no. of tiller per hill and days to heading on different rice genotypes grown under short day length condition in greenhouse at Tokyo University of Agriculture was presented in Table 1.2. There were significant differences among the tested genotypes for mean plant height under short day length condition in greenhouse. Mean plant height on PSM and Culture 340 was not significant different from one another and they were significantly taller than the other tested varieties. According to this data, it was found that mean plant height on PSM was significantly taller than the tested genotypes except culture 340 and the increase of plant height was seems be due to elongation of stem internodes. The rapid increase of plant length was anindication of changing vegetative to reproductive phaseof crop growth depending on the genotype (Krishnan et al., 2011).

Mean number of tiller (no.) in short day length condition for five rice varieties was significantly different. The number of tillers hill-1 were counted minimum to maximum. The maximum mean number of tillers hill-1 was observed in PSM (5.08) followed by culture 340(4.41). The minimum mean number of tillers hill-1 was obtained from IR 24 (3.50) followed by Thaingchung 65 (4.00) and IR 64 (4.33). Hussain, 2005 found that there was high competition among tillers in Japonica and indica varieties as compared to Nerica-4. In this study, it was observed that the maximum mean number of effective tillers hill was found in PSM seems be to due to their genetic variations and the minimum number was observed in IR 24 because it had the lowest mortality rate due to lower competition among tillers.

Days to heading showed significant difference among the tested varieties ranged between 50-92 days. The earliest heading variety was found in culture 340 (65 days) followed by IR64 (84 days), and the latest one was obtained in Paw San Hmwe (107 days) followed by IR 24 (90 days) and Thaichung65 (89 days). Days to heading of Paw San Hmwe were significantly later than the tested varieties. Zhou.et.al, 2011 reported that the heading date of some Indica and Japonica varieties was strongly influenced by their photoperiod sensitive gene in short day length. In my study, Paw San Hmwe was the latest variety among the tested variety because it had a perfect match gene in photoperiod sensitivity due to photoperiod sensitive variety. Even though there was short day treatment, days to heading of PSM were too late among the tested varieties. But, PSM from short day length was used as female parent for hybridization because it was synchronized with other three indica varieties (IR 24, Thaichung 65 and IR 64) from second time cultivation in a concrete tank of net house. 
Table 1.2:-Plant height, number of tiller per hill and days to heading under short day length condition in greenhouse at the Tokyo University of Agriculture

\begin{tabular}{|l|l|l|l|}
\hline Varieties & $\begin{array}{l}\text { Plant height } \\
\text { (Mean } \pm \text { SE) }\end{array}$ & $\begin{array}{l}\text { No. of tiller } \\
\text { per hill } \\
\text { (Mean } \pm \text { SE) }\end{array}$ & $\begin{array}{l}\text { Days to } \\
\text { heading } \\
\text { (Mean } \pm \text { SE) }\end{array}$ \\
\hline PSM & $111.16 \pm 1.45 \mathrm{a}$ & $5.08 \pm 0.50 \mathrm{a}$ & $107 \pm 1.00 \mathrm{a}$ \\
\hline Culture340 & $102.38 \pm 3.97 \mathrm{ab}$ & $4.41 \pm 0.55 \mathrm{a}$ & $65 \pm 1.00 \mathrm{~d}$ \\
\hline IR 24 & $90.95 \pm 1.62 \mathrm{bc}$ & $3.50 \pm 0.29 \mathrm{a}$ & $90 \pm 0.66 \mathrm{~b}$ \\
\hline Taichung65 & $93.71 \pm 0.95 \mathrm{~cd}$ & $4.00 \pm 0.25 \mathrm{a}$ & $89 \pm 0.66 \mathrm{~b}$ \\
\hline IR 64 & $82.06 \pm 1.28 \mathrm{~d}$ & $4.33 \pm 0.36 \mathrm{a}$ & $84 \pm 1.00 \mathrm{c}$ \\
\hline Pr>F & $<0.0001$ & $<0.0001$ & \\
\hline CV\% & 3.9 & 16.5 & $<0.0001$ \\
\hline
\end{tabular}

Means followed by the same letter are not significantly different at $5 \%$ level by Tukey'sStudentized Range (HSD) test

Effect of plant height, no. of tiller per hill and days to heading of five rice varieties under neutral day length and short day length condition:-

Mean plant height, no. of tiller per hill and days to heading of five rice varieties between neutral day length and short day length condition were shown in figure 1.1, 1.2 and 1.3. Mean plant height of PSM in neutral day length was taller than those in short day length. But, mean plant height of another four indica varieties in neutral day length was shorter than those in short day length. Result showed that plant height of PSM could be reduced due to short day length treatment. Similar result found that short day treatment could not only high plant height but also shorten 50\% flowering (Ganashen and Whittingtun, 1976 and Craufurd et.al., 2003).

Although mean no. of tiller per hill of culture 340 in neutral day length was lower than those in short day length, mean no. of tiller per hill of another tested varieties in neutral day length was higher than those in short day length. The reason for lower tiller number in short day length might be due to short day treatment (9 hour light and 15 hour dark) inhibits tiller multiplication. Similar result observed that short day treatment inhibited tillering in all the sowings (Misra and Khan, 1972).

Days to heading of all tested varieties in short day length was earlier than those in neutral day length. The short day treatment (9hour light and 15 hour dark) accelerates heading in all tested varieties compared to neutral day length. Morinaga et al., 1955 similarly observed that short day conditions accelerated inflorescence initiation, heading.

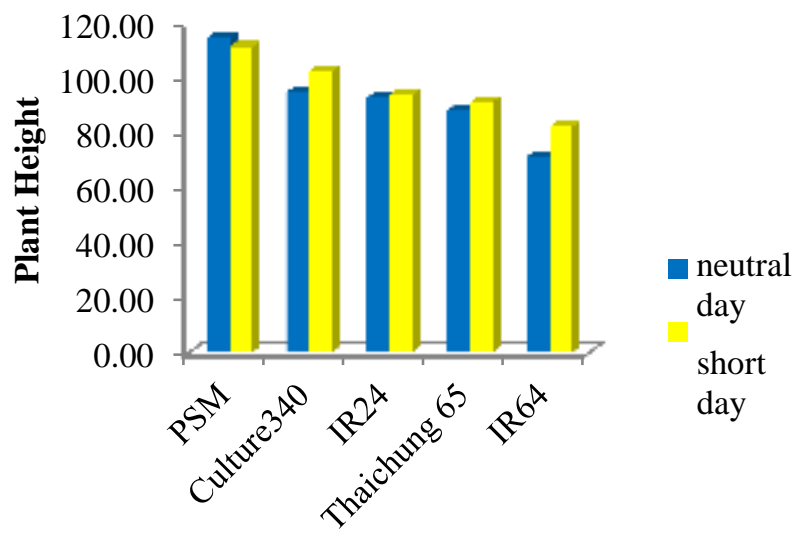

varieties

Figure 1.1:-Mean plant height affected by neutral day length and short day length on different rice varieties 


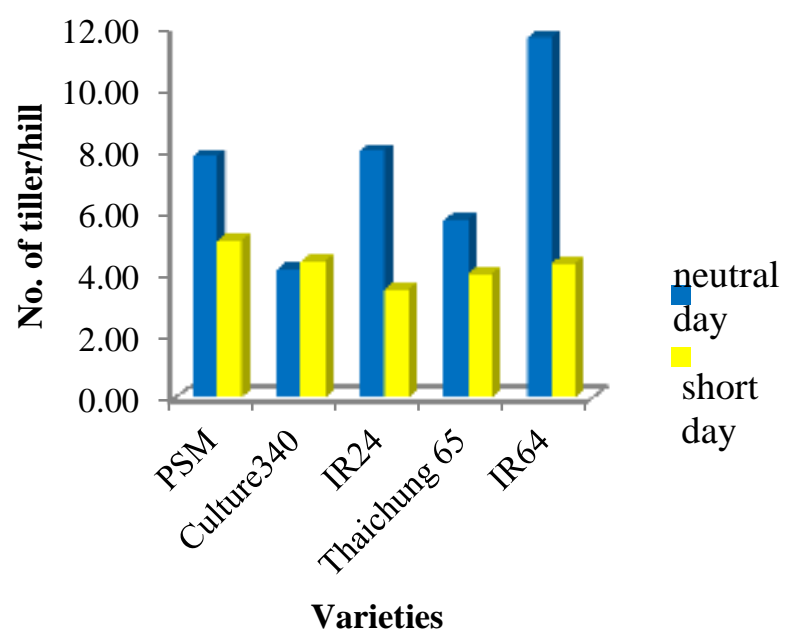

Figure 1.2:- Mean no. of tiller per hill affected by neutral day length and short day length on different rice varieties

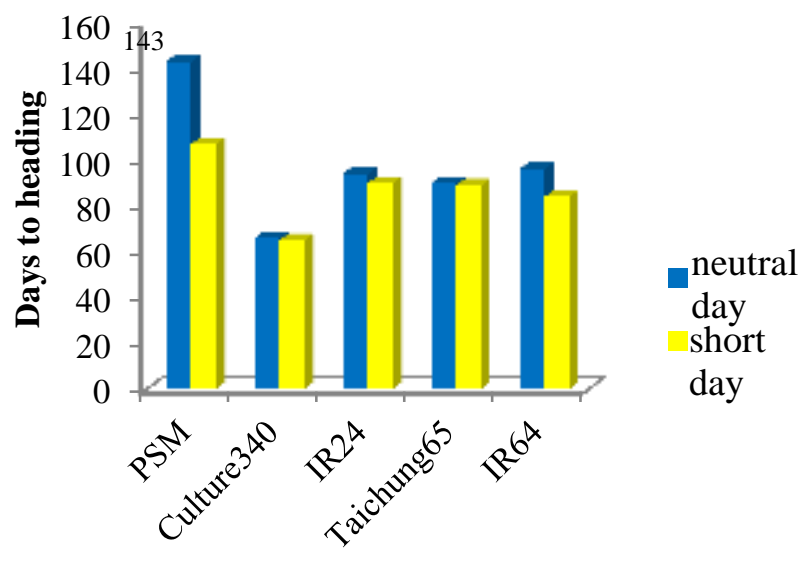

Varieties

Figure 1.3:-Days to heading affected by neutral day length and short day length on different rice varieties

\section{Comparison of crosses on hot water emasculation:-}

In this study, PSM from short day length used as female parent and hybridized with three indica rice varieties (IR 24, Thaichung 65 and IR 24) from second time cultivation in a concrete tank of net house because synchronization was found in short day length and concrete tank of net house.

Approximately 5 days after crossing, F1 seed set started on PSM x IR 24 and PSM x Thaichung 65 . F1 seed produced appeared to be light green colour on PSM x IR 24 and PSM x Thaichung 65. After 7 weeks, PSM x IR 24 and PSM x Thaichung 65 were fully matured. F1hybrid seed set percent on different crosses were shown in Table 1.3.

The cross ( PSM x IR 24) which was emasculated with hot water and pollinated by using 18 panicles for each pot obtained a high seed set (26\%). The F1 seed set of PSMx IR 24(26\%) was higher than PSM x Thaichung $65(21 \%)$. PSM x IR 64 did not get any other seed set. In this study, two of the crosses( PSM x IR 24 and PSM x Thaichung 65) made after hot water emasculation achieved $100 \%$ hybrid efficiency, but one cross (PSM x IR 64) (0\%) was completely unsuccessful. According to this study, pollen used for hybridization may be sterile and due to might be genetic differences. Causse and Ghesquiere, 1991 similarly reported pollination success was $0 \%$ and 5\% success in 
crosses between the two species, japonica and indica. Baldi, 1967 observed that there was low seed set after crossing depending on the genetic differences.

Table 1.3:-F1hybrids combinations and F1 seed set percent

\begin{tabular}{|l|l|}
\hline Crosses & $\begin{array}{l}\text { F1 seed set } \\
\text { Percent }(\%)\end{array}$ \\
\hline PSM x IR 24 & 26 \\
\hline PSM x IR 64 & 0 \\
\hline PSM x Thaichung 65 & 21 \\
\hline
\end{tabular}

\section{Conclusion:-}

Five rice varieties were cultivated by using neutral day length and short day length (9hours light and 15 hours dark) at 31.C to compare heading dates for synchronization. According to the present study, it could be concluded that in neutral day length, PSM was latest heading variety and it was highly sensitive to photoperiod. Culture 340 was the earliest varieties among the tested varieties and it could insensitive to highly photoperiod under neutral day length condition. Although IR 64 was shortest variety among the tested variety, tiller number was highest and the second latest variety in this study. IR 64, IR 24 and Thaichung 65 observed moderate photoperiod insensitive due to moderate heading date. In this experiment, plant height, number of tiller per hill and days to heading were significantly different from one another at 0.0001 probability level among the tested varieties under neutral day length condition.

In short day length, PSM was found the latest heading variety and it was highly sensitive to temperature (31.C) and photoperiod (9 hours light). Culture 340 was the earliest variety among the tested varieties and it could insensitive to temperature (31.C) and photoperiod (9 hours) because it is photoperiod insensitive variety under short day length condition. IR 64 was the second latest variety among the tested variety in this study. IR 24 and Thaichung 65 could moderate insensitive to photoperiod due to moderate flowering. Thus, it could response 9 hours light and 15 hours dark. The present study revealed that plant height, number of tiller per hill and days to heading were significantly different from one another at 0.0001 probability level among the tested varieties under short day length condition.

In this study, Culture 340 was earliest variety because of responsiveness between neutral day length and short day length condition. Therefore, Culture 340 was strongly insensitive to photoperiod under two conditions. Among the tested varieties, Paw San Hmwe (PSM) was latest variety due to low of responsiveness in both neutral day length and short day length conditions. But PSM could response short day treatment more than neutral day length. The present study revealed that although five rice varieties were cultivated to adjust synchronization under neutral day length and short day length for hybridization, synchronization for PSM and other four indica rice varieties was not found in both conditions because it might be different genetic variation on the tested varieties and sowing date of tested varieties was same under neutral day length and short day length condition. But 9 hour photoperiod is appropriate for synchronization of flowering affected in other four indica varieties ranging from very early to very late heading varieties. It was found that among the tested varieties; PSM was highly sensitive to high temperature and photoperiod in neutral day length due to not only flowering in short day condition but also photoperiod sensitive and late cultivar. But it was response to 9 hours light and 15 hours dark and flowered in short day length condition. In this study, PSM from short day length condition was used as doner parent for hybridization because it was synchronized with the other three indica varieties from cultivation of concrete tank in net house.

PSM (japonica) selected from short day length and three indica rice varities grown in concrete tank of net house were hybridized to observe F1 seed set by hot water emasculation method. In the two crosses (PSM x IR 24 and PSM x Thaichung 65), F1 seed set of PSM x IR 24 was better that of PSM x IR 64. In my present study, F1 seed set rate of PSM x IR 24 was at least 5\% more than PSM x Thaichung 65 as well as the maturity time of PSM x IR 24 was earlier than PSM $x$ Thaichung 65. PSM (japonica) is possible to cross to get F1 hybrid seeds with improved indica varieties such as IR 24 and Thaichung 65. Therefore, short day length condition ( 9 hour light and 15 hour dark) should be used for early heading compared to neutral day length condition especially in breeding program. The crosses (PSM x IR 24 and PSM x Thaichung 65) from this study should be used to get new rice varieties through several techniques. 


\section{References:-}

1. Abdul, R. A. 1969. Photoperiod and temperature effect on the growth and development of rice (Oryza sativa L.). The University of British Columbia. P. 71-72.

2. Baldi, G., 1967. Techniques and methods of artificial hybridization in rice. Selected seed XIII (1), 34-43.

3. Cause, M. and A. Ghesquiere, 1991. Prospective use of O. Longistaninata for rice breeding: In rice Genetics II Rice Research Institute. Phillippines. Pp. 81-89.

4. Craufurd, P. Q., I. E. Hauser, and M. Dingkuhn. 2003.Photothermal responses of $O$. sativa and $O$. glaberrimavarieties and interspecific progenies from West Africa. Field Crops Res, 83(3): 313-324.

5. Ganashan, P., and W. J. Whittington . 1976. Genetic analysis of the response to day length in rice. Euphytica, 25(1): 107-115.

6. Garner. W.W., and H.A. Allard. 1920. Effect of the relative length of day and night and other factors of the environment on growth and reproduction in plants. Agric Res, 18:553-606.

7. Hussain. S., T. Fujii, S. McGoey, M. Yamada, M. Ramzan, and M. Akmal. 2005. PARC Rice Programme Kala Shah Kaku, Lahore. JICA Training Center Tsukuba, Japan.

8. International Rice Research Institute (IRRI). 1976. Climate and rice. Los Banos, Philippines. IRRI: 418p.

9. Krishnan, P., B. Ramakrishnan, K.R. Reddy, and V.R. Reddy .2011. High-temperature effects on rice growth, yield, and grain quality. In Donald L. Sparks, Editor: Advances in Agronomy. 111, Academic Press Burlington. pp. 87-206.

10. Matsubayashi. M, R. Ito, T.Nomoto, T. Takase, and N. Yamada (eds.). 1965. Theory and practice of growing rice. Fuji Publishing Co., Tokyo.

11. Misra. G., and P.A. Khan . 1972. Interaction between short photoperiod and the month of sowing in a late winter indica rice. 137-147.

12. MOAI (Ministery of Agricultural and Irrigation). 2014. Myanmar Agriculture in Brief. Ministery of Agriculture and Irrigation, Myanmar.

13. Morinaga, T., H.Kurlyama and H. Kudo. 1955. Some experiments on photoperiodism in rice. Studies on rice breeding. (Jap.J. Breeding) 4:35-63.

14. Nareiso, J., and M. Hossain. 2002. World Rice Statistics. In, (IRRI).

15. Rjabov, I. E. 1941. Some aspects of photoperiodisms in rice Jarovizacija, no. $3: 48-55$.

16. Song., Y.L, Z.C. Gao and W.J. Luan. 2012. Interaction between temperature and photoperiod in regulation of flowering time in rice. Life Sciences, 3:24- 249.

17. Thein, M.S. 2011. Marginal land vs productive land: A case study on cultivating Myanmar local quality rice (Oryza sativa L.) morphotypes. Phetchaburi, Thailand: P148.LDD.

18. Win, U.K., 1991. A century of rice improvement in Burma. International Rice Research Institute, Manila, ISBN: 971-22-0024-8, Pages: 162.

19. Zhou, Z.L., L.Jiang, K. Liu, X. Da- yong, Z. hu- qu, and W. Jian- min. 2011. Crop genetics and germplasm enhancement, Nanjing agricultural university, Nanjing, rice science 2011, 18 (4): 287-296, china national research institude.

20. Zou, J.S., C.G.Lu, N.Hu, Y.Z.Li, and K.M. Yao. 2008. Studies on ecological adaptability and suitable planting area of a two-line hybrid rice, Liangyoupeijiu. SciAgric Sin, 41(11): 3563-3572. 